\title{
Corpus
}

$10 \mid 2011$

Varia

\section{Corpus de langue des signes : situer les biais des méthodes d'annotation et d'analyse}

Sign Language corpora: identifying the biases in annotation and analysis methods

Annelies Braffort, Emilie Chételat-Pelé et Jérémie Segouat

\section{OpenEdition}

Journals

Édition électronique

URL : http://journals.openedition.org/corpus/1992

DOI : $10.4000 /$ corpus. 1992

ISSN : $1765-3126$

Éditeur

Bases; corpus et langage - UMR 6039

Édition imprimée

Date de publication : 1 novembre 2011

Pagination : $25-40$

ISSN : 1638-9808

Référence électronique

Annelies Braffort, Emilie Chételat-Pelé et Jérémie Segouat, «Corpus de langue des signes : situer les biais des méthodes d'annotation et d'analyse », Corpus [En ligne], 10 | 2011, mis en ligne le 08 juin

2012, consulté le 08 septembre 2020. URL : http://journals.openedition.org/corpus/1992 ; DOI :

https://doi.org/10.4000/corpus.1992 


\title{
Corpus de langue des signes : situer les biais des méthodes d'annotation et d'analyse
}

\author{
Annelies BRAFFORT \\ LIMSI-CNRS (Orsay) \\ Emilie CHETELAT-PELE \\ Université de Provence (Aix-en-Provence) \\ Jérémie SEGOUAT \\ WebSourd (Toulouse)
}

\section{Introduction}

Les langues des signes (LS) sont les langues naturelles des communautés de personnes sourdes. Ces langues, dites visuogestuelles (émises par le corps et reçues via la vision), s'expriment dans l'espace devant le locuteur (appelé espace de signation), au moyen d'unités gestuelles ou " unités de sens ", composées de configurations de mains, de direction de regard, de mouvements du buste, d'expressions du visage, etc. réalisés simultanément.

Ce sont des langues dites "peu dotées » (Braffort \& Dalle 2011). Les études sur les LS sont assez récentes et les connaissances sur lesquelles il est possible de s'appuyer sont peu nombreuses (quand elles ne sont pas soumises à débat). Les LS ne disposent pas de système d'écriture, ce sont des langues de l'oralité : les études s'appuient donc sur des productions « orales» de LS, enregistrées sous forme de corpus vidéo (ou de capture de mouvement depuis peu).

Il existe encore peu de corpus et les outils permettant l'alignement de l'annotation et de la vidéo sont récents (Sloetjes \& Wittenburg 2008, Kipp à paraître, Hanke \& Storz 2008). L'analyse de corpus vidéo à l'aide de ces logiciels est maintenant systématiquement utilisée pour faire émerger les structures linguistiques des LS (De Beuzeville, Johnston \& Schembri 
2009 ; Johnston 2010 ; Efthimiou, Fotinea, Vogler, et al. 2009). Si un certain consensus existe dans la communauté scientifique sur le choix des éléments minimaux à annoter pour permettre un échange entre chercheurs (segmentation des unités de sens, attribution d'un identifiant, traduction approchée avec une langue écrite (Johnston 2008)), les objectifs sont très divers. Il peut s'agir de valider des modèles théoriques préexistants et les catégories associées (approche " basée corpus » (corpus-based)) ou de faire émerger de nouvelles catégories en dehors d'un cadre théorique bien marqué (approche «dirigée corpus» (corpusdriven)). Dans le premier cas, on recherche des structures connues appartenant à des modèles théoriques préexistants. L'analyse ne modifie pas les modèles en profondeur mais permet de valider ou affiner des connaissances. A l'inverse, la deuxième approche part de l'observation du corpus, puis produit les catégories théoriques à partir du comptage d'événements langagiers typiques et récurrents (Tognini-Bonelli 2001 ; Mayaffre 2005 ; Condamines 2005). Cette approche permet de faire émerger de nouveaux modèles.

Les deux approches sont susceptibles de générer des biais car il faut faire des choix, quant aux unités annotées, ou quant aux outils d'analyse utilisés pour faire émerger des catégories (lesquelles choisir d'étudier parmi celles existantes, ou sur quoi s'appuyer pour en proposer?). Plus généralement, il est important de bien identifier la part de subjectivité lors de l'annotation et les sources de biais potentielles en général, afin de relativiser les résultats des analyses de l'annotation et d'éviter de propager des erreurs issues des étapes initiales des études. De plus, identifier les biais méthodologiques potentiels peut permettre d'intégrer au plus tôt des processus de compensation ou de contrôle.

En tâchant de situer objectivement les choix effectués et les biais potentiels à chaque étape, cet article décrit les méthodologies que nous avons mises en place dans trois études, portant respectivement sur la synchronisation des composantes corporelles, le mouvement des sourcils et les clignements des yeux, puis la coarticulation. L'idée est de dresser un tour d'horizon des différents types de biais que nous avons pu détecter à 
Corpus de langue des signes : situer les biais

des méthodes d'annotation et d'analyse

ce jour, ainsi que les pistes pour y remédier, lorsque nous avons pu en proposer.

\section{Etude de la synchronisation des composantes corporelles}

Un des aspects les moins connus en LS est la manière dont se synchronisent les composantes corporelles, par exemple le regard et les mains, lors d'un pointage. Nous développons actuellement une méthode d'annotation et d'analyse permettant de déterminer des patrons de synchronisation des composantes présentes de manière systématique dans des structures linguistiques au niveau grammatical.

De nombreux articulateurs (mains, bras, buste, épaules, tête, regard, sourcils, joues, etc.) peuvent jouer un rôle dans la structure des énoncés. Afin de limiter la durée de la phase d'annotation (qui peut être considérable si l'on annote tous les articulateurs), nous avons fait une sélection parmi les articulateurs qui nous semblaient a priori les plus productifs lorsque le regard a une fonction liée à l'activation de locus dans l'espace de signation (Cuxac 2000). Ainsi, dans cette étude, nous avons choisi d'annoter uniquement l'activité des mains et des bras, ainsi que le regard.

Dans un premier temps, nous avons identifié les unités gestuelles (ou « signes »). Pour cela, nous nous sommes inspirés de la méthode proposée par Johnston (2011) pour la langue des signes australienne, que nous avons adaptée à nos observations. Chaque unité gestuelle est identifiée en fonction de son rôle linguistique (unité lexicale, pointage, description d'une relation spatiale, etc.) et de sa forme (celle de ces composantes corporelles). Ainsi, nous cherchons ici des catégories prédéfinies, même si ces catégories ont été affinées et modifiées pendant les premières phases d'annotation du corpus (approche de type « basée corpus »).

Dans un deuxième temps, nous avons annoté la direction du regard. Ici encore, nous avons utilisé et adapté les catégories proposées par Johnston, mais nous avons ajouté une piste d'annotation supplémentaire pour spécifier l'entité regardée dans le cas où le regard est dirigé vers l'espace de signation. Cela nous permet d'identifier le rôle linguistique du 
regard, qui peut être la création ou la réactivation d'un locus dans l'espace de signation selon Cuxac (2000). Pour cette étape, nous avons eu besoin d'interpréter les énoncés, afin de déterminer le rôle linguistique du regard. Il est même nécessaire d'interpréter l'ensemble du discours, car les phénomènes liés à l'utilisation de l'espace de signation ont une portée plus longue que l'énoncé.

Pour extraire les patrons de synchronisation entre les activités des différentes composantes corporelles, il est nécessaire d'analyser les relations temporelles entre ces activités. Cela revient à observer les relations temporelles entre les segments d'annotation pour les différentes pistes d'annotation (les rectangles horizontaux dans la figure 1). Pour cela, nous utilisons un langage de description qui permet d'exprimer des contraintes temporelles entre ces intervalles de temps, en termes de précédence, égalité, chevauchement (Filhol 2011a; Filhol 2011b).

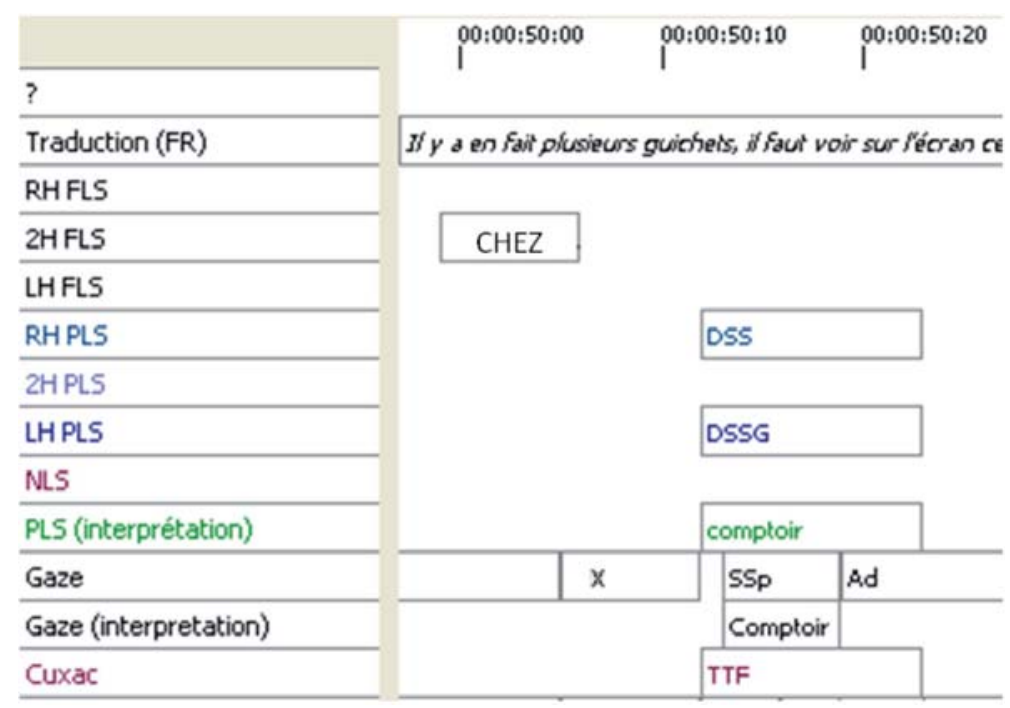

Figure 1. Extrait d'annotation avec le logiciel iLex, le temps s'écoule de gauche à droite 
Corpus de langue des signes: situer les biais des méthodes d'annotation et d'analyse

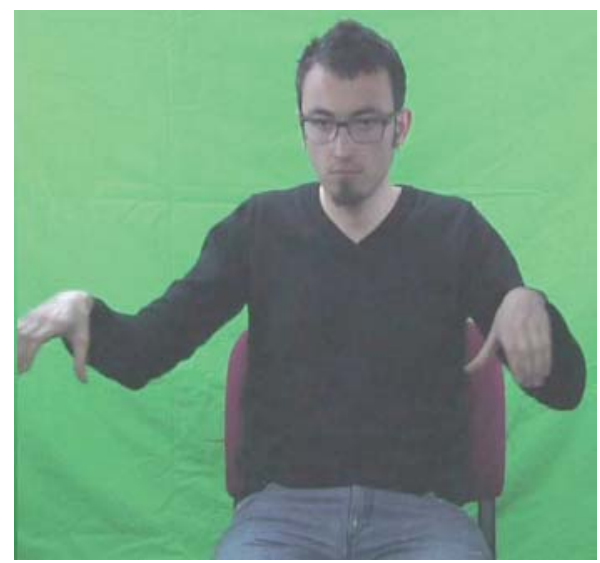

Figure 2. Unité gestuelle montrant un comptoir

Nous devons d'abord sélectionner les évènements à mettre en relation. Par exemple, nous avons étudié les relations entre les intervalles de temps relatifs à des évènements manuels et ceux durant lesquels le regard est dirigé vers une cible donnée sur un extrait de corpus de dialogue de LSF (Eftimiou et al. 2009). Ces relations s'expriment au niveau de l'énoncé et peuvent impliquer plusieurs signes. Dans ce cas, nous devons regrouper les signes en groupe de sens, ce qui requiert d'interpréter l'énoncé. Finalement, nous regroupons ou séparons les relations en fonction de leurs ressemblances et différences, ce qui permet de faire émerger des catégories.

Nous explicitons la méthode sur un exemple illustré figure 1. Cette figure montre un extrait d'annotation d'une structure gestuelle main / regard qui est employée pour décrire une entité (figure 2). La partie manuelle correspond à une structure iconique représentant un comptoir (dans le contexte d'une salle d'enregistrement dans un aéroport) tout en montrant sa forme (horizontale et allongée). Le regard accompagne cette activité manuelle. Les noms des pistes et les valeurs des attributs sont indiqués ci-dessous entre apostrophes. L'unité gestuelle a été catégorisée selon deux modèles théoriques : avec la valeur 'TTF' (pour Transfert de Taille et de Forme) selon les 
catégories de Cuxac (Cuxac 2000) et DS (pour Depicting Sign) selon les catégories de Johnston (Johnston 2011). Dans ce dernier modèle, la forme et le rôle de chaque main sont distingués ('DSS' pour la main droite qui trace la forme du comptoir - piste 'RH' - et 'DSSG' pour la main gauche qui sert de référent locatif stable - piste 'LH'). Le regard (piste 'Gaze') est dirigé vers l'espace de signation ('SSp') sur une zone que l'on a interprétée comme étant attribuée au comptoir (piste 'Gaze interpretation'). La relation temporelle que l'on observe entre les composantes manuelles et le regard est une inclusion: l'intervalle de temps pendant lequel le regard est en interaction avec la composante manuelle est inclus dans l'intervalle de temps des segments relatifs à la main droite et gauche. Nous cherchons à établir si c'est ce type de relation qui est le plus fréquent pour ce type de structure et si d'autres relations peuvent être observées (précédence, chevauchement, etc.) et dans quelle proportion. On obtient ainsi des patrons de synchronisations entre les différentes composantes corporelles qui forment un ensemble de catégories associées à des fonctions linguistiques (dans notre exemple : description et spatialisation d'une entité).

Afin de décider si un patron de synchronisation relève d'une catégorie plutôt que d'une autre, nous devons fixer un seuil de durée maximum au dessus duquel on considère que deux relations temporelles sont différentes. A minima, on peut prendre comme seuil une image, sachant que les vidéos comportent généralement 25 images par seconde.

Les différentes étapes sont susceptibles de générer des biais : choix des articulateurs annotés, identification des catégories des signes, interprétation de l'énoncé et sélection des relations. De plus, les règles de synchronisation en LS peuvent être assez flexibles. Si on fixe un seuil trop bas on risque de discriminer les phénomènes à un niveau trop fin et distinguer des relations qui sont en fait identiques. Enfin, les relations relevées entre deux intervalles temporels ne sont pas toujours pertinentes. Deux évènements se passant au même moment peuvent être indépendants ou synchronisés avec d'autres articulateurs ou d'autres évènements. Ils peuvent de ce fait fausser le comptage des relations. Tous ces facteurs peuvent amener à 
Corpus de langue des signes: situer les biais des méthodes d'annotation et d'analyse

distinguer trop de patrons, ou à séparer des évènements qui devraient appartenir au même patron.

Nous cherchons actuellement comment adapter notre protocole afin de corriger ces biais, en ajoutant des étapes de contrôle. La première étape consistera à annoter des composantes corporelles supplémentaires et faire varier le seuil permettant de distinguer les catégories. On pourra ensuite comparer le nombre et la représentativité des catégories et retenir le seuil qui nous semblera le plus adapté. Cela se fera de manière empirique dans un premier temps, avant de pouvoir déterminer des critères basés sur des données statistiques.

\section{Etude du mouvement des sourcils et des clignements des yeux}

Les mouvements des sourcils et des clignements des yeux ont été évoqués dans de nombreuses études qui soulignent leur importance en LS (Baker-Shenk 1985; Jouison 1995), mais leur analyse est récente et incomplète. Il est nécessaire d'analyser l'activité de ces composantes en interaction avec les autres composantes, manuelles ou non. Par exemple, nous souhaitons savoir quand une hausse de sourcils est nécessaire, quelle partie du sourcil doit être levée, sur quelle durée, etc. Nous avons mené une telle étude en suivant l'approche "dirigé corpus » (Chételat-Pelé \& Braffort 2010 ; Chételat-Pelé 2010).

Nous avons tout d'abord créé une méthodologie permettant d'annoter tous les mouvements perceptibles (et pas uniquement ceux connus pour leur pertinence). Nous avons choisi de décrire le mouvement qui conduit d'une posture à l'autre et pas la posture elle-même : ainsi, image par image, nous décrivons l'évolution d'un élément, par exemple l'extérieur du sourcil. Pour décrire ces mouvements, nous avons décomposé chaque sourcil en trois parties (extérieur, milieu et intérieur) en nous appuyant sur les travaux d'Ekman et Friesen (1978).

Pour annoter, nous avons créé un système symbolique permettant de prendre en compte l'amplitude d'un mouvement. Ainsi, nous distinguons trois amplitudes de mouvement: une standard, une faible et une forte (figure 3). 


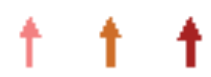

Figure 3. Symboles graphiques utilisés pour l'annotation des trois amplitudes de mouvement

Ces amplitudes ne sont pas utilisées pour caractériser des postures mais bien des mouvements d'une image par rapport à l'image précédente. Une posture peut donc être caractérisée par plusieurs amplitudes cumulées. Par exemple, si l'on regarde la posture indiquée par la flèche sur la figure 4, il s'agit d'une hausse élevée atteinte par une amplitude forte (cercle du bas), suivie d'une amplitude standard (cercle du haut).

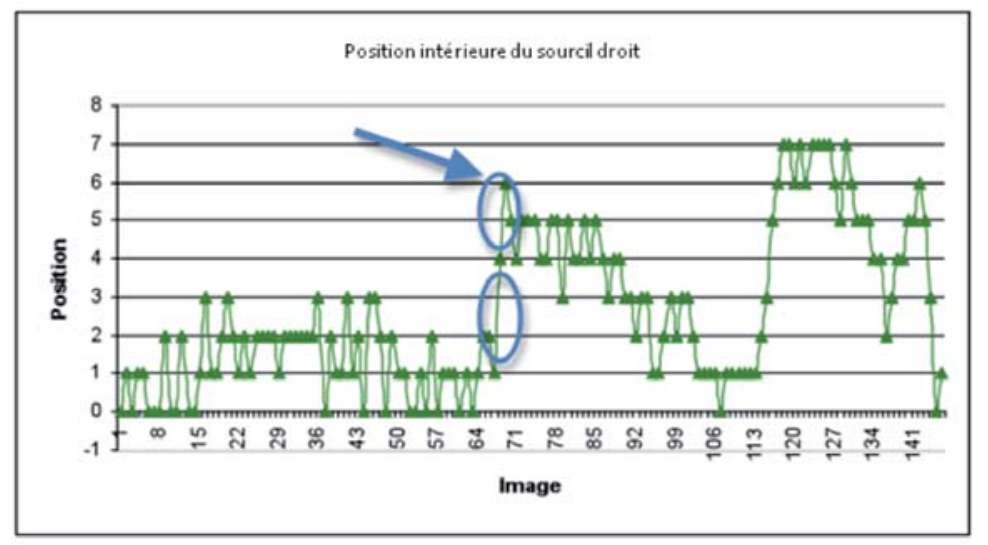

Figure 4. Position intérieure du sourcil droit

Cette annotation, qualitative, rend l'analyse plus aisée $\mathrm{du}$ fait de son caractère très visuel. Le fait $\mathrm{d}$ 'annoter image par image peut sembler excessif mais cela permet de diminuer la marge d'erreur en ne décrivant que de tous petits mouvements. Toutefois, cette annotation reste relativement subjective car l'amplitude d'un mouvement peut être perçue différemment d'une personne à l'autre.

Pour diminuer ce biais nous avons réalisé une deuxième annotation quantitative qui consiste en un positionnement de points dont nous récupérons les coordonnées, image par image, directement sur la vidéo. Les points sont positionnés sur chacune 


\section{Corpus de langue des signes : situer les biais des méthodes d'annotation et d'analyse}

des parties du sourcil (intérieur, extérieur et milieu) comme le montre la figure 5 .

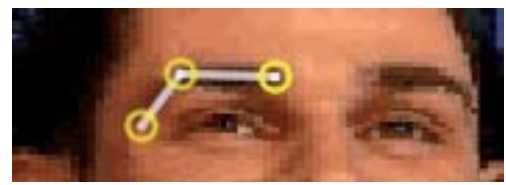

Figure 5. Emplacement des points d'intérêt sur le sourcil

Cependant, le positionnement manuel des points peut entraîner une grande variabilité aussi bien d'un annotateur à l'autre que chez un même annotateur. C'est pourquoi nous avons doublé les points des extrémités et triplé celui du milieu afin de réduire la marge d'erreur (figure 6).

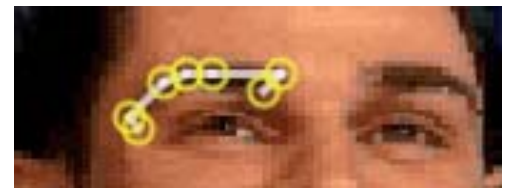

Figure 6. Ensemble des points annotés sur le sourcil

Le choix du nombre de points peut avoir influencé nos résultats. Ces derniers auraient sans doute été légèrement différents si nous avions positionné plus de points, avec l'inconvénient que des données plus nombreuses auraient sûrement augmenté le bruit. De même, si nous avions positionné moins de points, les données auraient probablement permis de mettre en avant uniquement les mouvements les plus marqués tout en engendrant moins de bruit. Nous avons cherché un compromis entre la précision des données et leur clarté. C'est pourquoi après quelques tests nous avons fixé le nombre de points à sept par sourcil.

La marge d'erreur due à l'annotateur humain pourra probablement diminuer si l'on utilise un logiciel de traitement d'images qui positionne les points automatiquement, encore qu'il faille évaluer le degré de précision de ce type d'outil qui est encore à l'état de prototype de laboratoire. Quoi qu'il en soit, il était nécessaire de commencer par une annotation manuelle afin 
de trouver les bonnes localisations et le bon nombre de points. De plus, nous disposons maintenant d'un corpus intégralement annoté à la main qui pourra servir de base de comparaison à des annotations automatiques lorsque les outils seront intégrés dans les logiciels d'annotation.

A partir des données obtenues, nous effectuons une série de calculs permettant d'obtenir une description numérique de chacun des mouvements.

Pour analyser l'ensemble des données obtenues, nous avons regroupé les mouvements en fonction de différents critères que nous avons testés jusqu'à trouver ceux qui semblaient les plus pertinents. Par exemple, nous avons regroupé les clignements en fonction de leur durée et de leur interaction avec les unités lexicales. Nous avons alors observé que certaines de nos catégories correspondaient à des catégories proposées par Cuxac (2000), tout en étant plus précises quant à la description des mouvements. Les catégories restantes semblent être de nouvelles catégories. A noter que nous avons fait le choix d'un travail fin et en profondeur, ce qui a eu pour conséquence une analyse d'un petit nombre de locuteurs. La méthodologie ayant été testée, il convient maintenant de l'automatiser autant que possible et de l'appliquer sur un corpus plus étendu afin de valider nos observations.

Lors d'une prochaine étude, nous pourrons probablement suivre une approche " basée corpus » afin de rechercher si les catégories que nous avons mises en évidence sont bien présentes. Ceci permettra d'augmenter les bases de données de chacune des catégories afin d'affiner l'analyse.

\section{Etude de la coarticulation}

La coarticulation est un phénomène encore peu étudié en LS, mais assez bien défini en Langue Vocale (LV). Bien qu'il subsiste encore quelques débats, la définition largement acceptée est qu'il s'agit de variations au niveau phonétique survenant lors de la mise en contexte d'éléments (phonétiques). Concrètement, en LV, la prononciation d'un élément sera différente selon que l'élément est isolé ou précédé et/ou suivi d'un autre élément, dans un mot, un énoncé, etc. (dans un contexte 
Corpus de langue des signes: situer les biais

des méthodes d'annotation et d'analyse

phonétique). Cette influence du contexte est réciproque, puisque l'élément influence lui aussi son environnement et entraîne des variations sur les autres éléments. Dans le cas des LS, la même définition s'applique, avec la différence notable qu'il n'existe pas de consensus quant à la définition de ce qui constitue le niveau phonétique (voir Boutora (2008) pour un état des lieux complet sur les différentes écoles de pensées).

Plus généralement, plusieurs courants de pensées définissent différemment ce qui constitue les différents niveaux linguistiques, ce qui constitue les unités de sens, ce qui fait partie du linguistique (Millet 2001 ; Boutora 2008). Dans notre étude, nous nous sommes positionnés dans le cadre théorique de Cuxac (2000), et nous avons considéré que le niveau phonétique est celui des "paramètres", qui peuvent être manuels (configuration, orientation, etc. des mains) ou non manuels (expression du visage, orientation du torse, direction du regard, etc.), et que le phénomène de coarticulation s'applique à ces paramètres, les faisant varier à l'image des variations de phones en LV. Le positionnement théorique linguistique en LS (comme pour toute langue) est donc très important puisqu'il a une influence sur la méthodologie mise en œuvre (les aspects étudiés sont différents d'un courant à l'autre), ainsi que sur la portée scientifique des conclusions.

Nous avons conçu et appliqué une méthodologie d'analyse de la coarticulation en LSF qui comporte plusieurs étapes, dont une d'annotation et une autre de traitement de ces annotations. Le choix des éléments annotés s'est fait au détriment d'autres : nous avons décidé, sur la base de critères linguistiques et d'adéquation avec l'objectif de notre étude, de nous intéresser aux configurations des mains et à la direction du regard. Cette décision a une influence sur nos conclusions, puisque nous nous sommes privés de la possibilité d'étudier les relations entre les éléments annotés et ceux non annotés, mais d'un autre côté cela nous a permis de nous focaliser sur un sous-ensemble d'éléments et de proposer un premier modèle. Concernant les valeurs utilisées pour ces annotations, nous avons choisi d'utiliser des images issues de captures d'écran de la vidéo, afin d'avoir des valeurs les plus objectives possibles, 
car elles reflètent exactement ce qui est dans la vidéo, sans passer par une schématisation ou une reformulation sous une autre forme graphique (figure 7). Ces images forment donc un vocabulaire contrôlé, ce qui assure une meilleure consistance de l'annotation, tout en permettant d'identifier directement la catégorie de l'élément à annoter.

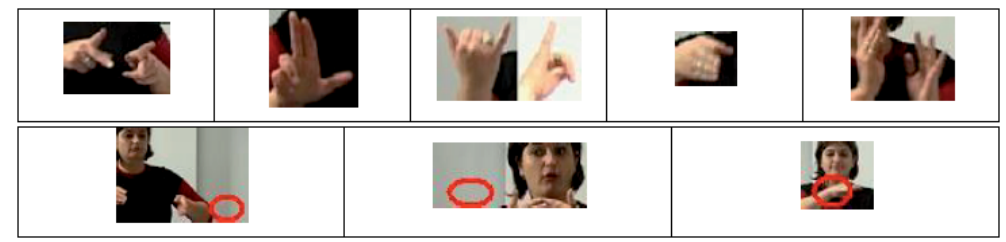

Figure 7. Annotations par capture d'écran

(en haut: annotations des configurations des mains ; en bas : annotations de la direction du regard)

L'intervention humaine ne permet pas de garantir une annotation identique tout au long du corpus : l'annotation manuelle, dans notre cas sur un support vidéo, entraîne une réflexion permanente sur la segmentation des éléments à annoter, la valeur à leur donner (puisque nous ne partions pas de catégories existantes, mais que nous avons créé nos propres valeurs) et la manière de les annoter. Dans notre étude, nous avons utilisé nos connaissances quant à la manière dont ont été conçus les énoncés pour déterminer le type de corrélations sur lesquelles appuyer nos résultats. Ils ont été produits dans un but de diffusion d'information: ils suivent une ligne éditoriale de présentation de l'information qui a un impact notamment sur les orientations, les emplacements des mains. De plus, les expressions du visage sont «contrôlées » dans le sens où il s'agit d'une diffusion d'information à l'échelon national, il ne nous a donc pas semblé pertinent de nous focaliser dans un premier temps sur cet aspect contraint dès le départ.

L'étape suivante est le traitement des annotations. Pour notre étude, nous avons adapté un algorithme d'alignement (développé initialement pour la bioinformatique) pour étudier les variations entre les annotations des formes isolées et celles des formes en contexte: ce procédé renvoie des informations 


\section{Corpus de langue des signes : situer les biais des méthodes d'annotation et d'analyse}

sur les similarités et la nature des différences sur les valeurs (addition, délétion ou substitution). Nous avons ainsi observé des relâchements et des tensions de configurations de mains, entre les expressions de signes isolés ou en contexte.

Le choix du traitement a un impact conséquent sur les résultats: par exemple, le traitement de ces annotations par recherche de corrélations temporelles (c'est-à-dire voir si les relations entre les éléments sont de type précédence, chevauchement, etc.), tel que cela a été fait dans l'étude présentée section 2, aurait pu permettre de spécifier plus précisément des ensembles de relâchements / tensions et de corréler ces constatations avec les variations d'autres paramètres (non étudiés dans le contexte de notre recherche).

Cette étude est «dirigée corpus »: bien que se positionnant dans un cadre théorique existant, elle n'a pas été basée sur un modèle de coarticulation. Il s'agissait de proposer un tel modèle à partir de l'analyse de ce corpus, et des récurrences d'évènements typiques langagiers.

\section{Conclusion}

D'un point de vue général, il semble important dans les études sur corpus d'anticiper les biais méthodologiques, afin de trouver des étapes compensatoires lorsque cela est possible, ou tout du moins de considérer les résultats d'analyse avec précaution. Cela nous semble un aspect particulièrement sensible pour les corpus de LS, pour lesquelles la constitution de corpus et l'annotation constituent des étapes très lourdes à mettre en œuvre et extrêmement longues. Ceci peut induire une tentation à généraliser trop vite à partir d'analyses partielles.

Dans les trois études présentées dans cet article, nous avons identifié et précisé des biais méthodologiques potentiels. Nous avons tenté, à chaque fois que cela était possible, d'ajouter dans la méthodologie des étapes de mesure, de validation ou d'analyse afin de limiter l'impact de ces biais. Parmi ces étapes supplémentaires, nous avons ajouté, selon le cas, des analyses sur de nouveaux critères, des mesures sur de nouveaux composants corporels. 
Il reste par ailleurs un aspect que nous n'avons pas encore évalué à une grande échelle. Il s'agit du taux d'accord inter-annotateur qui peut beaucoup varier selon les composantes annotées et la méthode utilisée.

Enfin, comme évoqué précédemment, un des principaux biais de l'annotation réside dans l'aspect manuel de la tâche, ce qui induit des risques d'imprécision ou de perte de cohérence d'ensemble. Les progrès réalisés en traitement d'images, reconnaissance automatique, ou encore l'utilisation de systèmes de capture de mouvements devraient dans les prochaines années modifier considérablement les usages et permettre de meilleures objectivité et précision. Cependant, les traitements ne réalisant que ce pour quoi ils ont été conçus, il restera toujours des précautions à prendre lors des étapes initiales d'analyse des corpus de LS.

\section{Références bibliographiques}

Allen J. F. (1983). « Maintaining Knowledge about Temporal Intervals », Communications of the ACM 26/11 : 832-843.

Baker-Shenk C. (1985). "The facial behavior of deaf signers : evidence of a complex language ", American Annals of the Deaf 130/4 : 297-304.

De Beuzeville L., Johnston T. \& Schembri A. (2009). « The use of space with indicating verbs in Australian Sign Language : A corpus-based investigation », Sign Language \& Linguistics 12/1:52-83.

Chételat-Pelé E. \& Braffort A. (2010). «Investigation et analyse des Gestes Non Manuels impliqués en LSF : le cas des clignements ", in Actes de la conférence Traitement Automatique des Langues Naturelles (TALN), atelier Traitement Automatique des Langues des Signes (TALS).

Chételat-Pelé E. (2010). Les Gestes Non Manuels en langue des signes française; Annotation, analyse et formalisation: Application aux mouvements des sourcils et aux clignements des yeux. Thèse de Doctorat de Sciences du langage, Université de Provence, Aix-en-Provence.

Condamines A. (2005). Sémantique et corpus. London : Hermes. 
Corpus de langue des signes : situer les biais
des méthodes d'annotation et d'analyse

Cuxac C. (2000). « La Langue des Signes Française : les voies de l'iconicité », Faits de Langues Vol. 15-16. Paris : Ophrys.

Efthimiou E., Fotinea S.-E., Vogler C., Hanke T., Glauert J., Bowden R., Braffort A., Collet C., Maragos P. \& Segouat J. (2009). "Sign Language Recognition, Generation, and Modelling: A Research Effort with Applications in Deaf Communication ", in Proceedings of the 5th International Conference on Universal Access in Human-Computer Interaction (UAHCI '09). Addressing Diversity. 5614/ 2009 : 21-30. Berlin : Springer-Verlag.

Ekman P. \& Friesen W. V. (1978). Facial Action Coding System. Mountain view, CA : Consulting Psychologists Press.

Hanke T. \& Storz J. (2008). «iLex - A database tool integrating sign language corpus linguistics and sign language lexicography », in Proceedings of the 6th International Conference on Language Resources and Evaluation. Paris : ELRA, pp. 923-926.

Johnston T. (2010). «From archive to corpus : transcription and annotation in the creation of signed language corpora ", International Journal of Corpus Linguistics 15/1 : 106-131.

Johnston T. (2011). Guidelines for annotation of the video data in the Auslan Corpus. Macquairie University, Sydney, Australia, http://www.auslan.org.au/media/auslan-video/ upload/attachments/AuslanCorpusAnnotationGuidelines15 March2011.pdf.

Jouison P. (1995). Ecrits sur la langue des signes française. Paris : L’Harmattan. Edité par B. Garcia.

Kipp M. (à paraître). «Multimedia Annotation, Querying and Analysis in ANVIL », in M. Maybury (ed.) Multimedia Information Extraction, Chapter 19, IEEE Computer Society Press.

Mayaffre D. (2005). «Rôle et place du corpus en linguistique. Réflexions introductives ", in Actes des Journées d'Etude TOUlousaines (JETOU 2005) Rôle et place des corpus en linguistique pp. 5-17, http://www.revue-texto.net/Corpus/ Publications/Mayaffre_Corpus.html. 
A. Braffort, E. Chételat-Pelé, J. SÉgOuAt

Segouat J., Choisier A. \& Braffort A. (2010). «Corpus de langue des signes: premières réflexions sur leur conception et leur représentativité », Travaux linguistiques $d u$ CerLiCO 23 (L'exemple et le corpus, quel statut ?) : 77-93.

Segouat J. (2010). Modélisation de la coarticulation en langue des signes française dans le contexte de la diffusion automatique d'informations en gare ferroviaire à l'aide d'un signeur virtuel. Thèse de Doctorat en informatique, Université de Paris XI, Orsay.

Sloetjes H. \& Wittenburg P. (2008). « Annotation by category ELAN and ISO DCR », in Proceedings of the 6th International Conference on Language Resources and Evaluation (LREC 2008). Paris : ELRA, pp. 923-926.

Tognini-Bonelli E. (2001). Corpus Linguistics at Work. Amsterdam: John Benjamins (Studies in Corpus Linguistics 6). 